\title{
Sufficient conditions for ergodic sensitivity
}

\author{
Xiong Wang ${ }^{\mathrm{a}, *}$, Xinxing $\mathrm{Wu}^{\mathrm{b}}$, Guanrong $\mathrm{Chen}^{\mathrm{c}}$ \\ a Institute for Advanced Study, Shenzhen University, Nanshan District Shenzhen, Guangdong, P. R. China. \\ ${ }^{b}$ School of Sciences, Southwest Petroleum University, Chengdu, Sichuan, 610500, P. R. China. \\ ${ }^{c}$ Department of Electronic Engineering, City University of Hong Kong, Hong Kong SAR, P. R. China.
}

Communicated by $\mathrm{X}$. Liu

\begin{abstract}
In this note, some sufficient conditions on the ergodic sensitivity of dynamical systems are obtained, improving the main results in [Q.-L. Huang, Y.-M. Shi, L.-J. Zhang, Appl. Math. Lett., 39 (2015), 31-34] and [R.-S. Li, Y.-M. Shi, Nonlinear Anal., 72 (2010), 2716-2720]. Moreover, it is proved that under these conditions, the second Lyapunov number of a dynamical system is equal to the diameter of its state space. (C)2017 All rights reserved.
\end{abstract}

Keywords: Sensitivity, ergodic sensitivity, Lyapunov number.

2010 MSC: 54H20, 74H65.

\section{Introduction}

A dynamical system is a pair $(X, T)$, where $X$ is a nontrivial compact metric space with a metric $\rho$ and $\mathrm{T}: \mathrm{X} \longrightarrow \mathrm{X}$ is a continuous map. Let $\mathbb{N}=\{1,2,3, \cdots\}$, and $\mathbb{Z}^{+}=\{0,1,2, \cdots\}$. For $\mathrm{U}, \mathrm{V} \subset \mathrm{X}$, define the return time set from $U$ to $V$ as $N(U, V)=\left\{n \in \mathbb{Z}^{+}: T^{n}(U) \cap V \neq \emptyset\right\}$.

Let $\mathcal{P}$ be the collection of all subsets of $\mathbb{Z}^{+}$. A collection $\mathscr{F} \subset \mathcal{P}$ is called a Furstenberg family if it is hereditary upwards, i.e., $F_{1} \subset F_{2}$ and $F_{1} \in \mathscr{F}$ imply $F_{2} \in \mathscr{F}$. A family $\mathscr{F}$ is proper if it is a proper subset of $\mathcal{P}$, i.e., neither empty nor the whole $\mathcal{P}$. It is easy to see that $\mathscr{F}$ is proper if and only if $\mathbb{Z}^{+} \in \mathscr{F}$ and $\emptyset \notin \mathscr{F}$.

For $A \subset \mathbb{Z}^{+}$, define

$$
\overline{\operatorname{dens}}(A)=\limsup _{n \rightarrow+\infty} \frac{1}{n}|A \cap[0, n-1]| \text { and } \underline{\operatorname{dens}}(A)=\liminf _{n \rightarrow+\infty} \frac{1}{n}|A \cap[0, n-1]| .
$$

Then, $\overline{\operatorname{dens}}(A)$ and $\underline{\operatorname{dens}}(A)$ are the upper density and the lower density of $A$, respectively.

A subset $A$ of $\mathbb{Z}^{+}$is syndetic if it has a bounded gap, i.e., if there is $N \in \mathbb{N}$ such that

$$
\{i, i+1, \cdots, i+N\} \cap A \neq \emptyset
$$

\footnotetext{
*Corresponding author

Email addresses: wangxiong8686@szu.edu.cn (Xiong Wang), wuxinxing5201314@163.com (Xinxing Wu), gchen@ee.cityu.edu.hk (Guanrong Chen)

doi:10.22436/jnsa.010.07.04
} 
for every $i \in \mathbb{Z}^{+}, A$ is thick, if it contains arbitrarily long runs of positive integers, i.e., for every $n \in \mathbb{N}$, there exists some $a_{n} \in \mathbb{Z}^{+}$such that $\left\{a_{n}, a_{n}+1, \cdots, a_{n}+n\right\} \subset A$. The set of all thick subsets of $\mathbb{Z}^{+}$and all syndetic subsets of $\mathbb{Z}^{+}$are denoted by $\mathscr{F}_{\mathrm{t}}$ and $\mathscr{F}_{\mathrm{s}}$, respectively.

For a Furstenberg family $\mathscr{F}$, a dynamical system is $\mathscr{F}$-transitive if $\mathrm{N}(\mathrm{U}, \mathrm{V}) \in \mathscr{F}$ for every pair of nonempty open subsets $\mathrm{U}, \mathrm{V} \subset \mathrm{X}$, and it is topologically strongly ergodic [6] if $\overline{\operatorname{dens}}(\mathrm{N}(\mathrm{U}, \mathrm{V}))=1$ for every pair of nonempty open subsets $U, V \subset X$. A dynamical system $(X, T)$ is topologically mixing if $\mathrm{N}(\mathrm{U}, \mathrm{V})$ is cofinite for every pair of nonempty open subsets $\mathrm{U}, \mathrm{V} \subset \mathrm{X}$.

The complexity of a dynamical system is a central topic of research since the term of chaos was introduced by Li and Yorke [13] in 1975, known as Li-Yorke chaos today. Another important feature of chaoticity is that orbits from nearby points start to diverge after finite steps. This notion, the "butterfly effect", has been widely studied and is termed as sensitive dependence on initial conditions (briefly, sensitivity), introduced by Auslander and Yorke [2] and popularized by Devaney [4]. More precisely, a dynamical system $(X, T)$ is sensitive if there exists $\varepsilon>0$ such that for any $x \in X$ and any $\delta>0$, there exist $y \in X$ with $\rho(x, y)<\delta$ and $n \in \mathbb{Z}^{+}$satisfying $\rho\left(T^{n}(x), T^{n}(y)\right)>\varepsilon$. The "size" of the set of all times where sensitivity emerges can be regarded as a measure of how sensitive a dynamical system is. Moothathu [14] initiated a preliminary study of various forms of sensitivity and proposed three stronger forms of sensitivity: syndetic sensitivity, cofinite sensitivity, and multi-sensitivity. Then, Li [11] introduced the concept of ergodic sensitivity, which is a stronger form of sensitivity, and showed that a strongly topologically ergodic system satisfying the large deviations theorem is ergodically sensitive. For more recent results on the notion of sensitivity, one is referred to $[8,14-22]$ and references therein.

For $\mathrm{U} \subset \mathrm{X}$ and $\varepsilon>0$, let

$$
M(U, \varepsilon)=\left\{n \in \mathbb{Z}^{+}: \operatorname{diam}\left(T^{n}(U)\right)>\varepsilon\right\},
$$

where $\operatorname{diam}(\cdot)$ denotes the diameter of a given set. It is easy to see that a dynamical system $(X, T)$ is sensitive if and only if there exists $\varepsilon>0$ such that for any nonempty open subset $U \subset X, M(U, \varepsilon) \neq \emptyset$. According to Moothathu [14] and Li [11], a dynamical system (X, T) is said to be

(1) ergodically sensitive, if there exists $\varepsilon>0$ such that for any nonempty open subset $U \subset X, \overline{\operatorname{dens}}(M(U, \varepsilon))$ $>0$;

(2) multi-sensitive, if there exists $\varepsilon>0$ such that for any $k \in \mathbb{N}$ and nonempty open subsets $\mathrm{U}_{1}, \cdots, \mathrm{U}_{\mathrm{k}} \subset$ $X, \bigcap_{i=1}^{k}\left\{n \in \mathbb{Z}^{+}: \operatorname{diam}\left(T^{n}\left(U_{i}\right)\right)>\varepsilon\right\} \neq \emptyset$;

(3) syndetically sensitive, if there exists $\varepsilon>0$ such that for any nonempty open subset $U \subset X, M(U, \varepsilon) \in$ $\mathscr{F}_{\text {s }}$;

(4) cofinitely sensitive, if there exists $\varepsilon>0$ such that for any nonempty open subset $U \subset X, M(U, \varepsilon)$ is cofinite.

Remark 1.1. By the definition of multi-sensitivity, it can be verified that a dynamical system is multisensitive if and only if there exists $\varepsilon>0$ such that for any $k \in \mathbb{N}$ and nonempty open subsets $\mathrm{U}_{1}, \cdots, \mathrm{U}_{\mathrm{k}} \subset$ $X, \bigcap_{i=1}^{k}\left\{n \in \mathbb{Z}^{+}: \operatorname{diam}\left(T^{n}\left(U_{i}\right)\right)>\varepsilon\right\}$ is an infinite set.

With respect to Devaney [4], a dynamical system $(X, T)$ is said to be chaotic in the sense of Devaney, if it satisfies the following three properties:

(1) $T$ is topologically transitive, i.e., for every pair of nonempty open sets $U, V \subset X$, there exists $n \in \mathbb{Z}^{+}$ such that $\mathrm{T}^{\mathrm{n}}(\mathrm{U}) \cap \mathrm{V} \neq \emptyset$.

(2) The set of periodic points of $T$ is dense in $X$.

(3) $T$ is sensitive.

Banks et al. [3] proved that the first two conditions (transitivity and dense periodic points set) imply the third condition-sensitivity, i.e., the above condition (3) is redundant. Later, Glasner and Weiss [5] obtained a stronger result which states that every topologically transitive map whose almost periodic points are dense in $X$ is sensitive. 
Very recently, Yin and Zhou [22] introduced the concept of ergodic chaos. According to them [22], a dynamical system $(X, T)$ is said to be ergodically chaotic, if $(X, T)$ is topologically ergodic and ergodically sensitive.

Kolyada and Rybak [10] recently introduced Lyapunov numbers to measure the sensitivity of dynamical systems. Define

$$
\begin{aligned}
& \mathbb{L}_{r}(T)=\sup \left\{\varepsilon: \quad \begin{array}{l}
\text { for every } x \in X \text { and every neighborhood } U_{x} \text { of } x, \text { there } \\
\text { exist } y \in U_{x} \text { and } n \in \mathbb{N} \text { such that } \rho\left(T^{n}(x), T^{n}(y)\right)>\varepsilon
\end{array}\right\}, \\
& \overline{\mathbb{L}}_{r}(T)=\sup \left\{\varepsilon: \quad \begin{array}{ll}
\text { for every } x \in X \text { and every neighborhood } U_{x} \text { of } x, \text { there } \\
\text { exists } y \in U_{x} \text { such that lim sup } \operatorname{sum}_{n \rightarrow+\infty} \rho\left(T^{n}(x), T^{n}(y)\right)>\varepsilon
\end{array}\right\},
\end{aligned}
$$

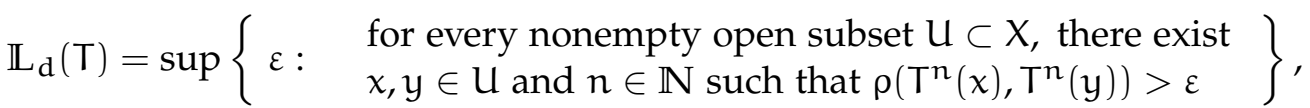

$$
\begin{aligned}
& \overline{\mathbb{L}}_{\mathrm{d}}(\mathrm{T})=\sup \left\{\begin{array}{ll}
\varepsilon: \quad \begin{array}{l}
\text { for every nonempty open subset } \mathrm{U} \subset \mathrm{X}, \text { there exist } \\
x, y \in U \text { such that } \lim \sup _{n \rightarrow+\infty} \rho\left(\mathrm{T}^{n}(x), T^{n}(y)\right)>\varepsilon
\end{array}
\end{array}\right\} .
\end{aligned}
$$

According to Kolyada and Rybak [10], $\mathbb{L}_{r}$ and $\mathbb{L}_{\mathrm{d}}$ are called the first Lyapunov number and the second Lyapunov number of $T$, respectively. In particular, they proved that for a minimal topologically weakly mixing system, all Lyapunov numbers are the same.

In 2002, Abraham et al. [1] gave some sufficient conditions for a measure-preserving transformation on a Borel probability measure space $(X, \mathcal{B}(X), \mu)$ to ensure the sensitivity property or confinite sensitivity property. In 2004, He et al. [7] proved that for a measure-preserving transformation $T$ on $(X, \mathcal{B}(X), \mu)$, if $\operatorname{supp}(\mu)=X$ and $T$ is weakly mixing, then $T$ is sensitive. They furthermore proved that this also holds for a measure-preserving semi-flow. In 2010, Li and Shi [12] showed that if a measure-preserving transformation on a Borel probability measure space with a full support is topologically strongly ergodic, then it is sensitive. Then, we [19] proved that every topologically strongly ergodic dynamical system satisfying the large deviations theorem is syndetically sensitive. Recently, Huang et al. [9] extended the main results obtained in $[1,6,7,12]$ as follows.

Theorem 1.2 ([9, Theorem 3.1]). Let $(X, T)$ be a dynamical system. If for every pair of nonempty open subsets $\mathrm{U}, \mathrm{V} \subset \mathrm{X}, \overline{\operatorname{dens}}(\mathrm{N}(\mathrm{U}, \mathrm{U}))+\underline{\operatorname{dens}}(\mathrm{N}(\mathrm{U}, \mathrm{V}))>1$, then $(\mathrm{X}, \mathrm{T})$ is sensitive.

\section{Main results}

In this note, we establish the following result to further extend Theorem 1.2.

Theorem 2.1. Let $(\mathrm{X}, \mathrm{T})$ be a dynamical system. If for every pair of nonempty open subsets $\mathrm{U}, \mathrm{V} \subset \mathrm{X}$, $\overline{\operatorname{dens}}(\mathrm{N}(\mathrm{U}, \mathrm{U}))+\underline{\operatorname{dens}}(\mathrm{N}(\mathrm{U}, \mathrm{V}))>1$ or $\underline{\operatorname{dens}}(\mathrm{N}(\mathrm{U}, \mathrm{U}))+\overline{\operatorname{dens}}(\mathrm{N}(\mathrm{U}, \mathrm{V}))>1$, then

(1) $(\mathrm{X}, \mathrm{T})$ is ergodically sensitive;

(2) $2 \mathbb{L}_{r}(T) \geqslant 2 \overline{\mathbb{L}}_{r}(T) \geqslant \mathbb{L}_{d}(T)=\overline{\mathbb{L}}_{d}(T)=\operatorname{diam}(X)$;

(3) $(X, T)$ is ergodically chaotic.

Proof. First, it is to claim that for any $A, B \subset \mathbb{Z}^{+}$, if $\overline{\operatorname{dens}}(A)+\underline{\operatorname{dens}}(B)>1$, then $\overline{\operatorname{dens}}(A \cap B)>0$.

Applying $\overline{\operatorname{dens}}(A)+\underline{\operatorname{dens}}(B)>1$ yields that there exist $\xi>1$ and an increasing sequence $\left\{n_{k}\right\}_{k=1}^{+\infty} \subset \mathbb{N}$ such that for any $k \in \mathbb{N}$,

$$
\frac{1}{n_{k}}\left|A \cap\left[0, n_{k}-1\right]\right|+\frac{1}{n_{k}}\left|B \cap\left[0, n_{k}-1\right]\right| \geqslant \xi .
$$

Suppose that the claim is false, i.e., $\overline{\operatorname{dens}}(A \cap B)=0$. Then

$$
\overline{\operatorname{dens}}(A \cup B)+\overline{\operatorname{dens}}(A \cap B) \leqslant 1,
$$


implying that there exists $N \in \mathbb{N}$ such that for any $n \geqslant N$,

$$
\frac{1}{n}|(A \cup B) \cap[0, n-1]|+\frac{1}{n}|(A \cap B) \cap[0, n-1]| \leqslant \frac{1+\xi}{2} .
$$

This is impossible, since

$$
\frac{1}{n}|A \cap[0, n-1]|+\frac{1}{n}|B \cap[0, n-1]|=\frac{1}{n}|(A \cup B) \cap[0, n-1]|+\frac{1}{n}|(A \cap B) \cap[0, n-1]|,
$$

holds for all $n \in \mathbb{N}$. This proves the claim.

Next, for any $0<\varepsilon<\operatorname{diam}(X)$, choose two points $z_{1}, z_{2} \in X$ such that $\mathrm{d}\left(z_{1}, z_{2}\right)>\varepsilon$. Then, there exists $\delta>0$ such that $\operatorname{dist}\left(\mathrm{B}\left(z_{1}, \delta\right), \mathrm{B}\left(z_{2}, \delta\right)\right):=\inf \left\{\rho(x, y): x \in \mathrm{B}\left(z_{1}, \delta\right), y \in \mathrm{B}\left(z_{2}, \delta\right)\right\}>\frac{\rho\left(z_{1}, z_{2}\right)+\varepsilon}{2}$, where $\mathrm{B}(z, \delta)=\{x \in X: \rho(x, z)<\delta\}$. Given any nonempty open subset $\mathrm{U} \subset X$, observing that $(X, T)$ is transitive, one has that there exists $k \in \mathbb{N}$ such that $W:=T^{-k}(U) \cap B\left(z_{1}, \delta\right) \neq \emptyset$. By the claim, it follows that

$$
\overline{\operatorname{dens}}\left(\mathrm{N}(\mathrm{W}, \mathrm{W}) \cap \mathrm{N}\left(\mathrm{W}, \mathrm{B}\left(z_{2}, \delta\right)\right)\right)>0 .
$$

For any $n \in N(W, W) \cap N\left(W, B\left(z_{2}, \delta\right)\right)$, there exist $x, y \in W$ such that $T^{n}(x) \in W \subset B\left(z_{1}, \delta\right)$ and $T^{n}(y) \in$ $\mathrm{B}\left(z_{2}, \delta\right)$. Then,

$$
\rho\left(T^{n}(x), T^{n}(y)\right)=\rho\left(T^{n-k}\left(T^{k}(x)\right), T^{n-k}\left(T^{k}(y)\right)\right)>\frac{\rho\left(z_{1}, z_{2}\right)+\varepsilon}{2}>\varepsilon .
$$

That is,

$$
\mathrm{N}(\mathrm{U}, \varepsilon) \supset \mathrm{N}(\mathrm{W}, \mathrm{W}) \cap \mathrm{N}\left(\mathrm{W}, \mathrm{B}\left(z_{2}, \delta\right)\right)-\mathrm{k} .
$$

This, together with [10, Proposition 2.1, Theorem 3.1], implies that $(X, T)$ is ergodically sensitive and $2 \mathbb{L}_{r}(T) \geqslant 2 \overline{\mathbb{L}}_{r}(T) \geqslant \mathbb{L}_{d}(T)=\overline{\mathbb{L}}_{d}(T)=\operatorname{diam}(X)$.

(3) It follows immediately from (1) of Theorem 2.1 and hypothesis.

Applying Theorem 2.1, [22, Theorem 7] can be improved to the following.

Corollary 2.2. Let $(X, T)$ be a topologically transitive system. If there exists a countable base $\left\{U_{i}\right\}_{i=1}^{\infty}$ of $X$ such that for any $i \geqslant 1, \underline{\text { dens }}\left(N\left(U_{i}, U_{i}\right)\right)>1 / 2$, then $(X, T)$ is ergodically chaotic. In particular, $(X, T)$ is Ruelle-Takens chaotic.

Similarly, the following result can be established.

Theorem 2.3. Let $(X, T)$ be a dynamical system. If for every pair of nonempty open subsets $\mathrm{U}, \mathrm{V} \subset \mathrm{X}$, $\mathrm{N}(\mathrm{U}, \mathrm{U}) \cap \mathrm{N}(\mathrm{U}, \mathrm{V}) \neq \emptyset$, then $(X, T)$ is sensitive and $2 \mathbb{L}_{r}(T) \geqslant 2 \overline{\mathbb{L}}_{r}(T) \geqslant \mathbb{L}_{d}(T)=\overline{\mathbb{L}}_{d}(T)=\operatorname{diam}(X)$.

Corollary 2.4. If a dynamical system $(X, T)$ is topologically strongly ergodic and satisfies dens $(\mathrm{N}(\mathrm{V}, \mathrm{V}))>0$ for every nonempty open subset $\mathrm{V} \subset \mathrm{X}$, then $(\mathrm{X}, \mathrm{T})$ is ergodically sensitive.

Corollary 2.5 ([12, Theorem 3.3]). Let $(X, \mathcal{B}(\mathrm{X}), \mu, \mathrm{T})$ be a measure-preserving system. If $\mathrm{T}$ is topologically strongly ergodic and $\operatorname{supp}(\mu)=X$, then $T$ is syndetically sensitive.

Proof. It follows immediately from Corollary 2.4, [14, Corollary 1], and the fact that $\mathrm{T}$ is $\mathscr{F}_{\mathrm{s}}$-transitive.

Remark 2.6. According to the proof of Theorem 2.1, it can be verified that Theorem 2.1 and Corollary 2.4 also hold for non-compact dynamical systems, semi-flows, and non-autonomous discrete dynamical systems. So, [9, Theorem 3.1, Theorem 3.2] and [12, Theorem 3.1] hold trivially.

Finally, an example is given to a dynamical system $(X, T)$ satisfying the assumptions of Theorem 2.1 with $\mathbb{L}_{r}(T)=\operatorname{diam}(X) / 2$. 
Example 2.7. Let $X=[0,1]$ and define $T: X \longrightarrow X$ by

$$
\mathrm{T}(x)= \begin{cases}4 x, & x \in[0,1 / 4], \\ -2 x+3 / 2, & x \in[1 / 4,1 / 2], \\ 2 x+1 / 2, & x \in[1 / 2,3 / 4], \\ -4 x+4, & x \in[3 / 4,1] .\end{cases}
$$

It can be verified that $(X, T)$ is topologically mixing, thus satisfies the assumptions of Theorem 2.1. Since for any $x \in X$ and any $n \in \mathbb{N},\left|T^{n}(1 / 2)-T^{n}(x)\right| \leqslant 1 / 2$, by Theorem 2.1, one has $\mathbb{L}_{r}(T)=\overline{\mathbb{L}}_{r}(T)=1 / 2$.

\section{Acknowledgment}

Xiong Wang was supported by the National Natural Science Foundation of China (No. 61601306) and Shenzhen Overseas High Level Talent Peacock Project Fund (No. 20150215145C).

Xinxing Wu was supported by the National Natural Science Foundation of China (No. 11601449) and the scientific research starting project of Southwest Petroleum University (No. 2015QHZ029).

Guanrong Chen was supported by the Hong Kong Research Grants Council under GRF Grant CityU 11201414.

\section{References}

[1] C. Abraham, G. Biau, B. Cadre, Chaotic properties of mappings on a probability space, J. Math. Anal. Appl., 266 (2002), 420-431. 1

[2] J. Auslander, J. A. Yorke, Interval maps, factors of maps, and chaos, Tôhoku Math. J., 32 (1980), 177-188. 1

[3] J. Banks, J. Brooks, G. Cairns, G. Davis, P. Stacey, On Devaney's definition of chaos, Amer. Math. Monthly, 99 (1992), 332-334. 1

[4] R. L. Devaney, An introduction to chaotic dynamical systems, Second edition, Addison-Wesley Studies in Nonlinearity, Addison-Wesley Publishing Company, Advanced Book Program, Redwood City, CA, (1989). 1, 1

[5] E. Glasner, B. Weiss, Sensitive dependence on initial conditions, Nonlinearity, 6 (1993), 1067-1075. 1

[6] R.-B. Gu, The large deviations theorem and ergodicity, Chaos Solitons Fractals, 34 (2007), 1387-1392. 1, 1

[7] L.-F. He, X.-H. Yan, L.-S. Wang, Weak-mixing implies sensitive dependence, J. Math. Anal. Appl., 299 (2004), $300-304$. 1

[8] W. Huang, P. Lu, X.-D. Ye, Measure-theoretical sensitivity and equicontinuity, Israel J. Math., 183 (2011), 233-283. 1

[9] Q.-L. Huang, Y.-M. Shi, L.-J. Zhang, Sensitivity of non-autonomous discrete dynamical systems, Appl. Math. Lett., 39 (2015), 31-34. 1, 1.2, 2.6

[10] S. Kolyada, O. Rybak, On the Lyapunov numbers, Colloq. Math., 131 (2013), 209-218. 1, 2

[11] R.-S. Li, The large deviations theorem and ergodic sensitivity, Commun. Nonlinear Sci. Numer. Simul., 18 (2013), 819-825. 1

[12] R.-S. Li, Y.-M. Shi, Several sufficient conditions for sensitive dependence on initial conditions, Nonlinear Anal., 72 (2010), 2716-2720. 1, 2.5, 2.6

[13] T. Y. Li, J. A. Yorke, Period three implies chaos, Amer. Math. Monthly, 82 (1975), 985-992. 1

[14] T. K. S. Moothathu, Stronger forms of sensitivity for dynamical systems, Nonlinearity, 20 (2007), 2115-2126. 1, 2

[15] X.-X. Wu, Chaos of transformations induced onto the space of probability measures, Internat. J. Bifur. Chaos Appl. Sci. Engrg., 26 (2016), 12 pages.

[16] X.-X. Wu, A remark on topological sequence entropy, Internat. J. Bifur. Chaos Appl. Sci. Engrg., (accepted).

[17] X.-X. Wu, G.-R. Chen, Sensitivity and transitivity of fuzzified dynamical systems, Inform. Sci., 396 (2017), 14-23.

[18] X.-X. Wu, P. Oprocha, G.-R. Chen, On various definitions of shadowing with average error in tracing, Nonlinearity, 29 (2016), 1942-1972.

[19] X.-X. Wu, X. Wang, On the iteration properties of large deviations theorem, Internat. J. Bifur. Chaos Appl. Sci. Engrg., 26 (2016), 6 pages. 1

[20] X.-X. Wu, J.-J. Wang, G.-R. Chen, F्F-sensitivity and multi-sensitivity of hyperspatial dynamical systems, J. Math. Anal. Appl., 429 (2015), 16-26.

[21] X.-X. Wu, L.-D. Wang, G.-R. Chen, Weighted backward shift operators with invariant distributionally scrambled subsets, Ann. Funct. Anal., 8 (2017), 199-210.

[22] J.-D. Yin, Z.-L. Zhou, Weakly almost periodic points and some chaotic properties of dynamical systems, Internat. J. Bifur. Chaos Appl. Sci. Engrg., 25 (2015), 10 pages. 1, 1, 2 